\title{
Digestion Mechanism Analysis of Total Arsenic Determination in Animal Origin Seafood by Atomic Fluorescence Spectrometry
}

\author{
Yong-Fu SHI ${ }^{1}$, Qian-Yun ZHAN ${ }^{1,2}$, Hui-Juan YU ${ }^{1}$, Run-Run GU ${ }^{1}$, \\ Dong-Mei HUANG ${ }^{1}$, Yuan WANG ${ }^{1}$, You-Qiong CAI ${ }^{1, *}$ \\ ${ }^{1}$ Fishery Products Quality Inspection and Test Center (Shanghai) of the Ministry of \\ Agriculture, Shanghai, China \\ ${ }^{2}$ Food Science and Engineering College, Ocean University of China, Qingdao, China \\ ${ }^{*}$ Corresponding author
}

Keywords: Digestion Mechanism, Total Arsenic, Seafood, Atomic Fluorescence Spectrometry.

\begin{abstract}
In the present study, canned crab and canned tuna were taken as the quality control samples, and microwave digestion combined with wet-digestion was estimated as the optimum digestion method for animal origin seafood after comparing with the microwave digestion. The quality control samples were digested under microwave first, and then $1 \mathrm{~mL} \mathrm{HClO}_{4}$ and $2 \mathrm{~mL} \mathrm{H}_{2} \mathrm{SO}_{4}$ were added to the digestion solution for wet digestion. Atomic Fluorescence Spectrometry (AFS) was adopted to determine the concentration of total arsenic in quality control samples. The result obtained is close to the assigned value with high accuracy. The relative error ranges from $1.8 \%$ to $2.1 \%$. The present study elaborates the experimental phenomena and conversion mechanism of different existing forms of arsenic in details during wet-digestion under $\mathrm{HNO}_{3}-\mathrm{HClO}_{4}-\mathrm{H}_{2} \mathrm{SO}_{4}$ system. And it is effective and convenient to control the time node by analyzing the experimental phenomena which is conductive to ensure the accurate results.
\end{abstract}

\section{Introduction}

Arsenic and its compounds which have been identified as carcinogens by International Agency for Research on Cancer (IARC) are widespread in nature [1]. They mainly include Arsenate (AsV), Arsenite (AsIII), Monomethylarsenic (MMA), Dimethylarsenic (DMA), TrimethylarsineOxide (TMAO), Arsenobetaine (AsB), Arsenoc-holine (AsC), and Arsenosugars (AsS) [2].

Marine organisms which contain high level of arsenic compounds are the main sources of arsenic from daily dietary intake[3]. With the improvement of people' $s$ living standard, the total consumption of aquatic products and processed aquatic products have increased significantly. Therefore, the determination of total arsenic is crucial for the quality control of animal origin seafood [4]. However, the existing forms of arsenic are pretty complicated and there are a large proportion of $\mathrm{AsB}$ and $\mathrm{AsC}$ in seafood [5,6].The application of different acid and the control of time node during the digestion are important for the conversion of total arsenic into inorganic arsenic $[7,8]$. In addition, the digestion level of samples strongly influences the determine results of total arsenic [9,10]. The Chinese national standard GB/T 5009.11-2003 is the valid national standard of determination of total arsenic and in organic arsenic executed currently. Previous research results have indicated that the parallelism and accuracy of the standard cannot meet the requirements of analysis [11]. 
The present study took the canned crab and canned tuna as the quality control samples, and found that microwave digestion combined with wet-digestion was the optimum digestion method under $\mathrm{HNO}_{3}-\mathrm{HClO}_{4}-\mathrm{H}_{2} \mathrm{SO}_{4}$ system. This study elaborated the experimental phenomena and conversion mechanism of different existing forms of arsenic in details during wet-digestion so that it was effective and convenient to control the time node by observing the experimental phenomena. Thus, this study was not only conductive to ensure the accurate results of determination of total arsenic in animal origin seafood, but also provided references for the digestion method of other sources of food.

\section{Experimental}

\section{Materials and Instrument}

Materials: quality control samples were all purchased from FAPAS. The content of total arsenic in canned crab and canned tuna were $11.2 \mathrm{mg} / \mathrm{kg}$ and $1.354 \mathrm{mg} / \mathrm{kg}$ respectively. Arsenic standard solution $(1000 \mu \mathrm{g} / \mathrm{mL})$ was obtained from Shanghai Institute of Measurement and Testing Technology.

Reagent and solutions: nitric acid was purchased from J.T.Baker. Sulphuric acid, perchloric acid, sodium hydroxide, sodium borohydride and thiocarbamide with high purity were all obtained from Sinopharm Chemical Reagent Co., Ltd. Deionised water which was from a Milli-Q water purification system was used for the preparation of all solutions.

Instrument: microwave digestion device was purchased from Milestone. Atomic fluorescence spectrometer (AFS-9230) was purchased from Beijing Titan instrument Co., Ltd. Electric heating panel was obtained from LabTech.

\section{Method}

Microwave Digestion. Initially, $0.2 \pm 0.05 \mathrm{~g}$ canned crab and $1 \pm 0.05 \mathrm{~g}$ canned tuna, respectively, were weighed in digestion vessels, then $6 \mathrm{~mL}$ nitric acid was added. After 12 hours, $2 \mathrm{~mL}$ of hydrogen peroxide was added. When the foam disappeared the microwave digestion procedure was carried out according to the following program(Table 1).Then the digestion vessels were placed on the electric heating panel to evaporate the digestion solution to $0.1-0.2 \mathrm{~mL}$. After cooling to room temperature, the digested samples were diluted in water up to $25 \mathrm{~mL}$. The $4 \mathrm{~mL}$ samples obtained were combined with $1 \mathrm{~mL} 10 \%$ thiocarbamide and final volume was made up to $10 \mathrm{~mL}$ with deionized water. Total arsenic of the samples was analyzed by AFS.

Table 1. Digestion program of microwave

\begin{tabular}{|c|c|c|}
\hline Procedure & Temperature ${ }^{\circ} \mathrm{C}$ & Run time $\min$ \\
\hline 1 & up to 200 & 15 \\
\hline 2 & 200 & 30 \\
\hline 3 & cool down & 100 \\
\hline
\end{tabular}

Microwave Digestion Combined with Wet-digestion. After microwave digestion, sample solutions were transferred into the $50 \mathrm{~mL}$ erlenmeyer flasks to cool down. $1 \mathrm{~mL}$ perchloric acid and $2 \mathrm{~mL}$ sulfuric acid were added, then the erlenmeyer flasks were placed on the electric heating panel to heat. Setting to $200^{\circ} \mathrm{C}$, perchloric acid volatilized into white smoke. When the white smoke disappeared, the temperature was set up to $350^{\circ} \mathrm{C}$ to volatilize sulfuric acid into white smoke. After cooling to room temperature, the samples were diluted in water up to $50 \mathrm{~mL}$. The $2 \mathrm{~mL}$ samples obtained 
were combined with $1 \mathrm{~mL} 10 \%$ thiocarbamide and $7 \mathrm{~mL}$ deionised water to determine the total arsenic using AFS.

Calibration Procedure. The external calibration technique was followed for the quantitative analysis of the samples. 200ng/mL Arsenic standard solutions were prepared by dilution of the stock solutions with $1 \%$ (w/w) HNO3. Afterward, 1mL10\% thiocarbamide were added and the solutions were diluted in water up to $10 \mathrm{~mL}$. Then the standard solution obtained was diluted automatically by AFS. The calibration curves were built on 6 different concentrations, namely 1.0, 2.0, 5.0, 10.0, 15.0, 20.0ng/mL.

\section{Results and Discussion}

\section{Analysis of Digestion Mechanism}

AsS, which predominantly found in algae, is accumulated in the body of animal origin seafood because of intaking algae [12]. Report found that AsS had high stability due to no significant changes occurred after treated at $100^{\circ} \mathrm{C}$ for $10 \mathrm{~min}$ [13]. AsB separated from lobster in 1977 is the main compound of arsenic form in animal origin seafood [14]. It can stay stable at $4^{\circ} \mathrm{C}$ for many years [15]. Besides, animal origin seafood has a portion of $\mathrm{AsC}$ which is not very stable. AsB and $\mathrm{AsC}$ will convert into TMAO and DMA later when heated in the nitric acid solution. DMA is a stable organic arsenic compound. Previous research showed that DMA is stable within $200^{\circ} \mathrm{C}$, while it will completely convert into inorganic arsenic at $300^{\circ} \mathrm{C}$ for $90 \mathrm{~min}$ [16].Therefore, the conversion is essential for the determination of total arsenic. Fig. 1 shows the conversion process.

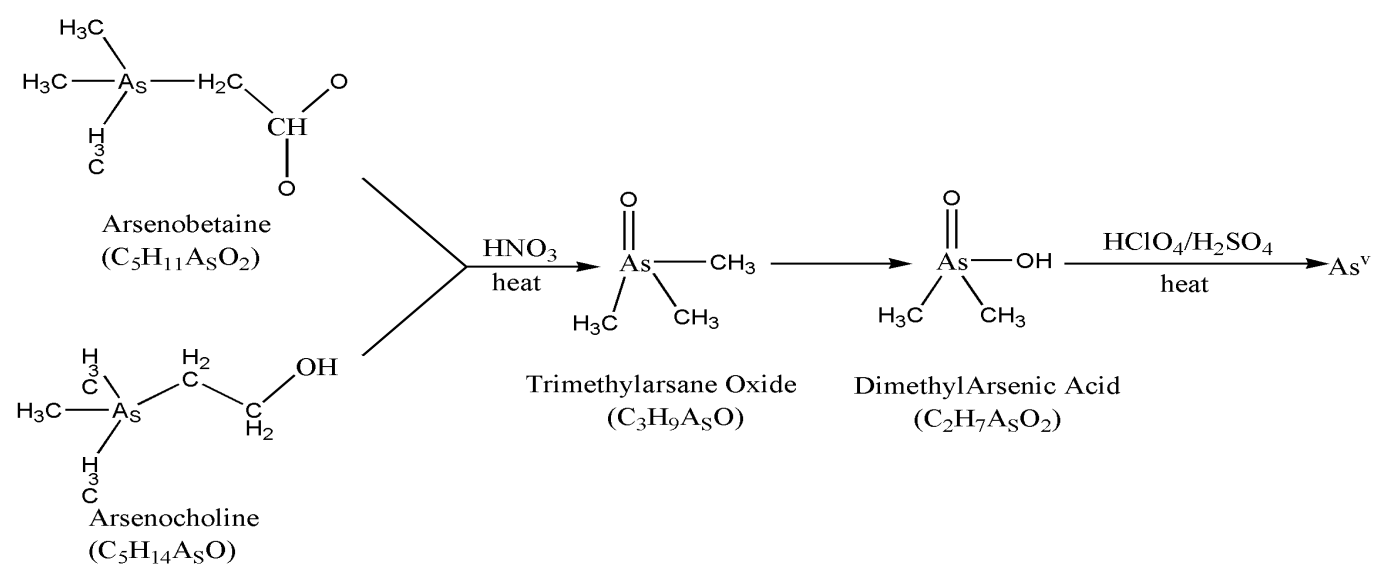

Figure 1. Digestion mechanism of Arsenobetaine (AsB) and Arsenocholine (AsC)

\section{Judgement of Phenomena and Control of the Time Node}

After microwave digestion which added nitric acid and hydrogen peroxide, rufous gas $\left(\mathrm{NO}_{2}\right)$ was releaed from the digestion vessels $\left(4 \mathrm{HNO}_{3}=4 \mathrm{NO}_{2} \uparrow+\mathrm{O}_{2} \uparrow+2 \mathrm{H}_{2} \mathrm{O}\right)$. Most of $\mathrm{AsB}$ and $\mathrm{AsC}$ have converted into DMA except the inorganic arsenic compounds. Then the digestion solutions were transferred into the $50 \mathrm{~mL}$ erlenmeyer flasks. $1 \mathrm{~mL}$ perchloric acid and $2 \mathrm{~mL}$ concentrated sulfuric acid were added, then the erlenmeyer flasks were placed on the electric platen to heat . Setting to $200^{\circ} \mathrm{C}$, perchloric acid volatilized into white smoke. This phenomenon means a majority of different forms of arsenic compounds have converted into inorganic arsenic. When the white smoke disappeared, warming up to $350^{\circ} \mathrm{C}$ (boiling point of sulphuric acid is $338^{\circ} \mathrm{C}$ ). The color of digestion solution turned chartreuse due to chlorine which was formed by the decomposition of perchloric acid at high temperature dissolved in water 
$(4 \mathrm{HClO} 4=2 \mathrm{H} 2 \mathrm{O}+7 \mathrm{O} 2 \uparrow+2 \mathrm{Cl} 2 \uparrow)$. Then the color changed transparent, which was the signal of perchloric acid decomposed and volatilized thoroughly. Continue heating, white smoke of sulphuric acid rose gradually and disappeared slowly, and the whole process lasted around 5 minutes. At this moment, the different arsenic form of animal origin seafood have converted into inorganic arsenic completely.

Three phenomena occurred when animal origin seafood were digested under the of $\mathrm{HNO} 3-\mathrm{HClO} 4-\mathrm{H} 2 \mathrm{SO} 4$ system. Firstly, perchloric acid volatilized into white smoke. Secondly, the color of digestion solutions changed from chartreuse to transparent. Thirdly, white smoke of sulphuric acid rose. After the three steps, the different arsenic forms have converted into inorganic arsenic completely. Thus, the judgement of phenomena and the control of time node assured the accuracy of the determination results.

\section{Calibration Curve}

Fig. 2 shows the regression equation and fluorescence intensity for standard solution of different concentration. Linear correlation coefficient is 0.9999 .

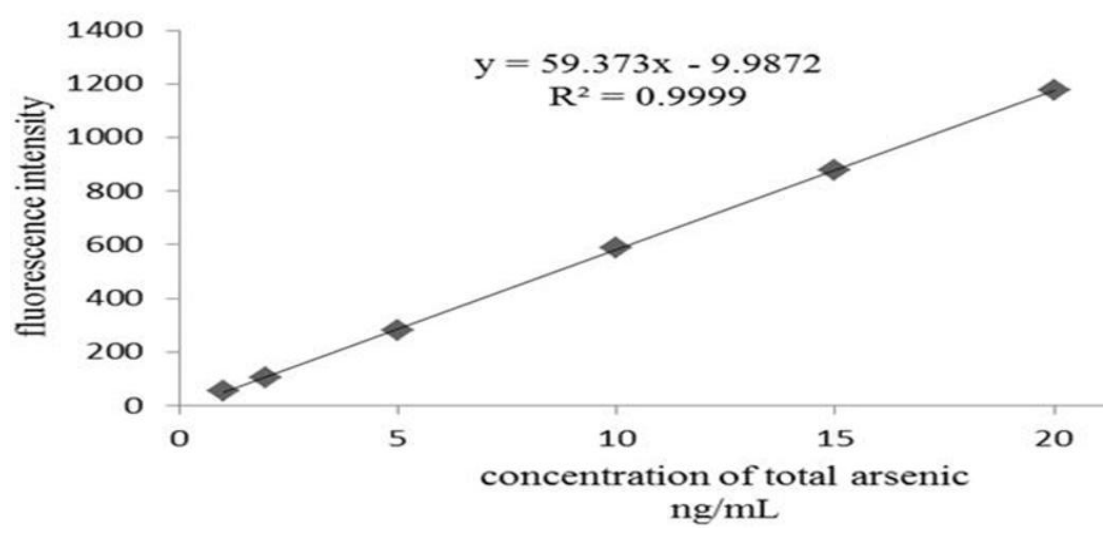

Figure 2. Standard curve and regression equation

\section{Results Analysis of Quality Control Samples}

The determination results of canned crab using microwave digestion and microwave digestion combined with wet-digestion are showed in Table 2.

Table 2. Results of different pretreatment $(n=4)$

\begin{tabular}{cccc}
\hline Digestion means & $\begin{array}{c}\text { Determination } \\
\text { valuemg/kg }\end{array}$ & $\begin{array}{c}\text { Reference value } \\
\mathrm{mg} / \mathrm{kg}\end{array}$ & $\begin{array}{c}\text { Relative error } \\
\%\end{array}$ \\
\hline Microwave digestion & 2.8 & 11.2 & 75 \\
\hline $\begin{array}{c}\text { Microwave digestion } \\
\text { combined with wet-digestion }\end{array}$ & 10.8 & 11.2 & 3.6 \\
\hline
\end{tabular}

The results obtained in this study showed that determination value of two pretreatments were $2.8 \mathrm{mg} / \mathrm{kg}$ and $10.8 \mathrm{mg} / \mathrm{kg}$ respectively. Comparing with microwave digestion, it was obvious that microwave digestion combined with wet-digestion which recovery rate is $96.4 \%$ could meet the analytical demand.

Using microwave digestion combined with wet-digestion, the total arsenic of quality control samples were determined, as presented in Table 3. 
Table 3. Testing results of canned fishery product pretreated by microwave and wet digestion

\begin{tabular}{lccc}
\hline Sample name & Determination valuemg/kg & Reference valuemg/kg & Relative error \% \\
\hline Canned crab & 10.92 & 11.2 & 2.1 \\
\hline Canned tuna & 1.469 & 1.354 & 1.8 \\
\hline
\end{tabular}

Obviously, the determination results of canned crab and canned tuna which were the representatives of animal origin seafood were highly close to the assigned value. Consequently, this microwave digestion combined with wet-digestion method developed and verified for determination of total arsenic was proved to be effective. It saves time and requires minimal amount of chemicals comparing with wet-digestion.

\section{Conclusions}

Previous researches have reported that the biggest problem which lead to inaccurate results of determination is the incomplete digestion due to the existence of arsenic compounds' different forms during the determination for the total arsenic in animal origin seafood [17,18]. In addition to the Chinese national standard (GB/T 5009.11.2003), the Chinese Specification for Marine Monitoring(GB 17378.6-2007) regulates that the digestion of marine organisms should use $\mathrm{HNO}_{3}-\mathrm{HClO}_{4}$ system [19]. Owing to the various kinds of animal origin aquatic products, and their different content of $\mathrm{AsB}$ and $\mathrm{AsC}$, the digestion using $\mathrm{HNO}_{3}-\mathrm{H}_{2} \mathrm{O}_{2}$ or $\mathrm{HNO}_{3}-\mathrm{HClO}_{4}$ may be applicable for the products which contain extremely little AsB and AsC. While samples could be thoroughly digested to get the accurate total arsenic concentration by using $\mathrm{HNO}_{3}-\mathrm{HClO}_{4}-\mathrm{H} 2 \mathrm{SO}_{4}$ as described even if the AsB and AsC of samples can not be certained. Meanwhile, the present study elaborated the experimental phenomena in details during wet-digestion under $\mathrm{HNO}_{3}-\mathrm{HClO}_{4}-\mathrm{H} 2 \mathrm{SO}_{4}$ system. It was not only conductive to ensure the accurate results of determination of total arsenic in animal origin seafood, but also provided references for the digestion of other source of food.

\section{Acknowledgement}

This study was supported by the Special Research Fund for National Non-profit of East China Sea Fishery Research Institute (NO.2011M12) and Agriculture Industry Research Special Funds for Public Welfare Projects (201503108-EC-1).

\section{References}

[1] J.H. Sun, L.Z. Wang, H.S. Lin, Hydride generation-atomic fluorescence spectrometry for inorganic arsenic inspection in fresh fishery products, J. Fisheries Science. 29 (2010) 366-368.

[2] W.H. Li, Y.H. Liu, Analysis of arsenic speciation in algae by high performance chromatography coupled inductively coupled plasma-mass spectrometry, J. Analysis Laboratory. 3 (2012) 74-78.

[3] M. Ana , C. Anica, S.T. Janja, M. Darja, K. Mladen, M. Marika, S. Katia, S. Zdravko, B. Fabio, H. Milena, Mercury, Arsenic and Selenium exposure levels in relation to fish consumption in the Mediterranean area, J. Environmental Research .120 (2013) 7-17.

[4] K.A. Francesconi,Arsenic species in seafood:origin and human health implications, J. Pure \& Applied Chemistry. 82 (2010) 373-381. 
[5] W.P. Norwood, U. Borgmann, D.G. Dixon, Saturation models of Arsenic, Cobalt, Chromium and manganese bioaccumulation by Hyalella Azteca, J. Environmental Pollution. 143 (2006) 519-528.

[6] S.T. Liu, T.C. Jiang ,P. Luo, D.S. Mo, J. Li, Z..X. Long, Q. Tang, H.Q. Ni, Determination and evaluation of heavy metal elements in seafood of Chinese northern Bay, J. Modern Food Science and Technology .29 (2013) 853-857.

[7] S.T. Liu, T.C. Jiang, J.M. Li, P. Luo, Y. Tao, Y.C. Song, Determination of Arsenic Speciation in Seafood by HPLC-AFS , J. Chemical Analysis and Meterage. 22 (2013) $11-14$.

[8] X.J.Yang, W. Huang, T. Lin, Y.G. Yu, J.E. Zhao, Determination of heavy metal contents in foodstuffs by three pretreatment methods, J. Modern Food Science and Technology. 24 (2008) 1051-1054.

[9] G.D. Yang, J.H. Xu, J.P. Zheng, X.Q. Xu, W. Wang, L.J. Xu, G.N. Chen, F.F. Fu, Speciation analysis of arsenic in Mya arenaria Linnaeus and Shrimp with capillary electrophoresis-inductively coupled plasma mass spectrometry, J. Talanta.78 (2009) 471-476.

[10] Z.Z. Qian, F.F. Luo, S.C. Zhu, Y. Hong, Progress on the Determination of Arsenic in Aquatic Products , J. Guangzhou Chemical Industry .40 (2012) 14-16.

[11] Y. Wu , W.L. Shuai, D.Z. Chen, Study on impact of different pretreatments on result of determination of total arsenic in aquatic products, J. Guangzhou Chemistry Industry. 38 (2010) 206-207.

[12] Y. Nishizawa, Arsenic and its toxicity in marine algae, J. Food development. 8 (1985) 35-38.

[13] C. Wei, W.H. Li, C. Zhang, M. Van Hulle, R. Cornelis, X.R. Zhang, Safety evaluation of organoarsenical species in edible porphyra from the China Sea, J. Agricultural and Food Chemistry. 51 (2003) 5176-5182.

[14] F.H. Chen, X.R. Zhang, Arsenicbetaine in marine animals, J. Marine Environmental Science. 12(1993):58-67.

[15] L. Zhang , Y.N. Wu, Y.F. Zhao, Study on the stability of different forms of arsenic compounds and the sample pretreatment technology, J. Foreign Medical Sciences(Section of Hygiene).34 (2007) 238-244.

[16] C. Lu, L.P. Liu, H.R. Dong, X.W. Li, Analysis of five arsenic speciations by High Performance Liquid Chromatography and Inductively Coupled Plasma Mass Spectrometry, J. Instrumental Analysis. 29 (2010) 465-468.

[17] W. Goessler, M. Pavkov, Accurate quantification and transformation of Arsenic compounds during wet ashing with Nitric Acid and microwave assisted heating, J. The Analyst. 128 (2003) 796-802.

[18] Y. Wang, Z.B. Yuan, Microwave digestion -HGAFS determination of microamount of arsenic in Oyster Shell, J. Analysis Laboratory. 22 (2002) 81-83.

[19] K. Wang , Q.S. Gao, Determination of trace arsenic in seafood with microwave digestion-atomic and fluorescence spectrometry, J. Modern Food Science and Technology .25 (2009) 848-851. 